Research article

\title{
Epidemiological features of traumatic spinal cord injury in Chongqing, China
}

\section{Guang-Zhi Ning, Zhi-Ping Mu, Lei Shangguan, Yu Tang, Chang-Qing Li, Zheng- Feng Zhang, Yue Zhou}

Department of Orthopedics, Xinqiao Hospital, the Third Military Medical University, Chongqing, Peoples Republic of China

Objective: To describe the epidemiological characteristics and trends of traumatic spinal cord injury in Chongqing, China.

Study design: Hospital-based retrospective research.

Setting: Xinqiao Hospital, Chongqing.

Methods: We reviewed medical records of 554 patients with traumatic spinal cord injury (TSCI) admitted to Xinqiao Hospital from 2009 to 2013. Variables included gender, age, marital status, etiology, occupation, time of injury, level of injury, and severity of injury, the length of hospital stay, and treatment.

Results: The mean age of patients with TSCI was $45.6 \pm 13.8$ years, and the male/female ratio was 4.33:1. 94.2\% (522 patients) of all patients with TSCI were married. Falls, comprising low falls and high falls ( $10.8 \%$ and $50.9 \%$, respectively), were the leading reason for a hospital visit. And the second reason was MVCs (21.8\%). The most common injury site was the cervical spinal cord, accounting for nearly more than $54 \%$. The length of hospital stay ranged between 1 and 219 days (mean: 28.3 days). The proportions of complete tetraplegia, incomplete tetraplegia, complete paraplegia and incomplete paraplegia were $17.1 \%, 37.8 \%, 22.2 \%$, and $22.9 \%$, respectively. Conclusion: The result revealed that the proportion of males was higher, as well as falls and MVCs were the first two main reasons, with older mean age of patients with TSCI than other countries. The occupations with highest risk for TSCI were peasants and laborers. All of these results prompted that preventive methods should be based on the characteristics of different type of patients with TSCI.

Keywords: Traumatic spinal cord injury, TSCI, Epidemiology, Prevention, High-risk occupations for SCI

\section{Introduction}

Traumatic spinal cord injury (TSCI) is a devastating condition that can cause serious dysfunction in sensation, motor ability, and autonomic function. TSCI results in a significant burden to both the individual and society that includes a negative influence on quality of life (QoL) and substantial economic costs to the healthcare system. ${ }^{1}$ Currently, there is no effective restorative cure for TSCI; thus, primary prevention is the best strategy.

The estimated annual incidence of TSCI varies from 10.4 per million to 83 per million in North America

Correspondence to: Zheng-Feng Zhang and Yue Zhou, Department of Orthopedics, Xinqiao Hospital, Xinqiao Street, Shapingba District, Chongqing 400037, Peoples Republic of China. Email: zhouyue_xinqiao@ 163.com

Guang-Zhi Ning and Zhi-Ping Mu contributed equally to this research. Zheng-Feng Zhang and Yue Zhou were both the Corresponding authors. and Western Europe. ${ }^{2,3}$ Among developed countries, there is up-to-date information on the incidence of TSCI. Noe et al. reported that the overall annual TSCI incidence during the study period, 1990-2012, was 10.2 per million person-years in Denmark. ${ }^{4}$ Koskinen et al. reported that the mean annual incidence of TSCI in Finland was 25.1 per million, and in the hospital districts of the SCI centers, the incidence was even higher, at 38.1 per million. ${ }^{5}$ In a review on developing countries, Robert et al reported that Saudi Arabia had a high incidence rate of SCI (38-62.37 per million) compared with most developed countries. ${ }^{6}$ RahimiMovaghar et al. summarized the incidence of SCI in developing countries and found a rate of $25.5 /$ million/ year, which ranged from 2.1 to $130.7 /$ million/year. ${ }^{7}$

The land mass area of China is the third largest in the world, and China is the world's most populous nation. In China, researchers have performed few studies 
about the epidemiology of TSCI, reporting an annual incidence rate of 23.7 per million population in Tianjin, ${ }^{8} 60.6$ per million in Beijing. ${ }^{9}$ There have also been a few hospital-based studies focused on epidemiological features rather than on the annual incidence of TSCI, such as Anhui ${ }^{10}$ and Tianjin ${ }^{11}$ Because each locale has its own epidemiological disease characteristics, it is of great importance to conduct such epidemiological research at the local population level. Additionally, significant variation in the prevalence of TSCI exists among geographic regions. As is well known, Chongqing is the largest and most populous area among the People's Republic of China's four provincial-level municipalities. However, at present, very little is known and reported about the prevalence of SCI in Chongqing. The goals of the present study were to explore the epidemiological features of patients with TSCI in Chongqing and to help to establish the best allocation of medical resources, in order to ease financial and social burdens.

\section{Methods}

To our knowledge, a regional population-based registry system of total TSCI has not yet been developed in Chongqing; thus, we retrospectively reviewed the medical records during 2009-2013 in Xinqiao Hospital, using the International Classification of Diseases Version 10 (ICD-10) and diagnostic code of TSCI. At first, 610 cases were identified, and demographic characteristics and epidemiological features were collected, including sex, age, marital status, occupation, etiology, neurological level of injury, American Spinal Injury Association impairment scale (ASIA grade), severity of injury, death, cause of death, concomitant injuries, the time from trauma to hospital admission, the length of hospital stay and treatment (conservative treatment or surgery). The inclusion criteria for the study were spinal cord injuries or cauda equina injuries, caused by trauma that originally occurred in Chongqing, and patients who were admitted to Xinqiao Hospital, Chongqing at the time of trauma. Exclusion criteria were vertebral fractures and intervertebral disc disease in the absence of TSCI, fatally injured persons and emergency department patients who were never hospitalized. Medical records with uncertain diagnoses and incomplete medical records were also excluded. After excluding the above situations ( 50 vertebral fractures and intervertebral disc disease in the absence of TSCI; 5 medical records with uncertain diagnosis; 1 incomplete medical records), a total of 554 cases were identified.

In the present study, like many other research, ${ }^{8,10,12}$ the cases were divided into six age groups with 15 year intervals: $0-15,16-30,31-45,46-60,61-75$, and $\geq 76$ years. Marital status was recorded as "married" or "unmarried"; possibly "divorced" and "widowed" were recorded as "unmarried", as medical records were often not detailed about marital status. The etiologies included motor vehicle collisions (MVCs), falls (low fall and high fall, $1 \mathrm{~m}$ as the boundary. For low fall, height $<1 \mathrm{~m}$; for high fall, height $\geq 1 \mathrm{~m}$ ), ${ }^{11}$ collision of head against objects, injuries caused by falling objects, injuries caused by machinery, sports, injuries caused by another person, and massage. Occupation included the unemployed, the retired, peasants, laborers, office-clerks, teachers, students and others. The neurological level of injury included cervical, thoracic, lumbar, and sacral segments. The injury severity and the motor and sensory functions below the injury segment were assessed using the ASIA grade.

Quantitative data processing was performed with SPSS version 19.0 (IBM, Armonk, NY, USA) statistical software. Categorical and continuous data are reported as proportions and means, respectively.

\section{Results}

\section{Sex and age}

Finally, the data from the 554 TSCI cases were collected. As shown in Table 1, the number of patients with TSCI increased over the 5 years of study (2009: 79, 2010: 90, 2011: 105, 2012: 128, and 2013: 152). Among 554 patients with TSCI identified, 450 were male and 104 were female, and the male/female ratio was 4.33:1. The oldest patient was 81 years old, and the youngest was 4 years old, with a mean age of $45.6 \pm 13.8$ years (males $45.7 \pm 13.3$ years, females $45.4 \pm 15.7$ years). Furthermore, the age group 31-45 years was the largest $(36.3 \%)$, followed by $46-60$ years $(33.8 \%)$. The count of patients with TSCI during 2009-2013 is shown in Fig. 1.

\section{Marital status}

As shown in Table 1, 94.2\% (522) of all patients with TSCI were married. The percentage of unmarried patients was 5.8\% (32), including the divorced and widowed and patients without any recorded detailed marital status.

\section{Occupations}

In the data collection process, we found that the occupations of patients with TSCI included the unemployed (134), peasants (177), laborers (131), office-clerks (20), the retired (11), teachers (2), students (10) and others (69). As shown in Table 1, peasants, laborers and the unemployed were the top three high-risk TSCI groups, 
Table 1 Characteristics of patients with TSCI from 2009 to 2013

\begin{tabular}{|c|c|c|c|c|c|c|}
\hline Years & 2009 & 2010 & 2011 & 2012 & 2013 & Total \\
\hline Count & 79 & 90 & 105 & 128 & 152 & 554 \\
\hline \multicolumn{7}{|l|}{ Age } \\
\hline $0-15$ & 0 & 2 & 0 & 5 & 0 & 7 \\
\hline $16-30$ & 11 & 10 & 12 & 19 & 19 & 71 \\
\hline $31-45$ & 34 & 39 & 43 & 42 & 43 & 201 \\
\hline $46-60$ & 23 & 24 & 33 & 40 & 67 & 187 \\
\hline $61-75$ & 11 & 15 & 17 & 20 & 21 & 84 \\
\hline$\geq .76$ & 0 & 0 & 0 & 2 & 2 & 4 \\
\hline Average age & 44.34 & 44.51 & 46.38 & 44.96 & 46.97 & 45.62 \\
\hline \multicolumn{7}{|l|}{ Sex } \\
\hline Male & 61 & 68 & 92 & 105 & 124 & 450 \\
\hline Female & 18 & 22 & 13 & 23 & 28 & 104 \\
\hline \multicolumn{7}{|l|}{ Marital status } \\
\hline Married & 74 & 82 & 104 & 118 & 144 & 522 \\
\hline Unmarried & 5 & 8 & 1 & 10 & 8 & 32 \\
\hline \multicolumn{7}{|l|}{ Occupation } \\
\hline Laborer & 7 & 15 & 23 & 46 & 40 & 131 \\
\hline Peasant & 26 & 30 & 42 & 36 & 43 & 177 \\
\hline Unemployed & 18 & 22 & 16 & 37 & 41 & 134 \\
\hline Office-clerks & 0 & 3 & 5 & 6 & 6 & 20 \\
\hline Retired & 2 & 1 & 3 & 2 & 3 & 11 \\
\hline Teacher & 0 & 1 & 0 & 0 & 1 & 2 \\
\hline Student & 0 & 3 & 0 & 3 & 4 & 10 \\
\hline Other & 7 & 9 & 13 & 11 & 15 & 69 \\
\hline \multicolumn{7}{|l|}{ Etiology } \\
\hline MVCs & 23 & 19 & 22 & 31 & 26 & 121 \\
\hline Low fall & 4 & 9 & 9 & 17 & 21 & 60 \\
\hline High fall & 38 & 48 & 53 & 57 & 86 & 282 \\
\hline Falling objects & 8 & 14 & 20 & 18 & 13 & 73 \\
\hline Collision of head & 1 & 0 & 0 & 0 & 0 & 1 \\
\hline Machine & 3 & 0 & 0 & 0 & 2 & 5 \\
\hline Sports & 1 & 0 & 1 & 0 & 1 & 3 \\
\hline Hyper-traction & 0 & 0 & 0 & 2 & 0 & 2 \\
\hline Other & 1 & 0 & 0 & 3 & 3 & 7 \\
\hline
\end{tabular}

Abbreviations: MVCs, motor vehicle collisions; TSCI, traumatic spinal cord injury.

accounting for $31.9 \%, 23.6 \%$, and $24.2 \%$ of the observations, respectively.

\section{Etiology}

The final data revealed that falls, comprising low falls and high falls (10.8\% and 50.9\%, respectively), were the leading reason for a hospital visit in our study. The second-leading cause was MVCs (21.8\%), followed by injury from falling objects as the third-most common

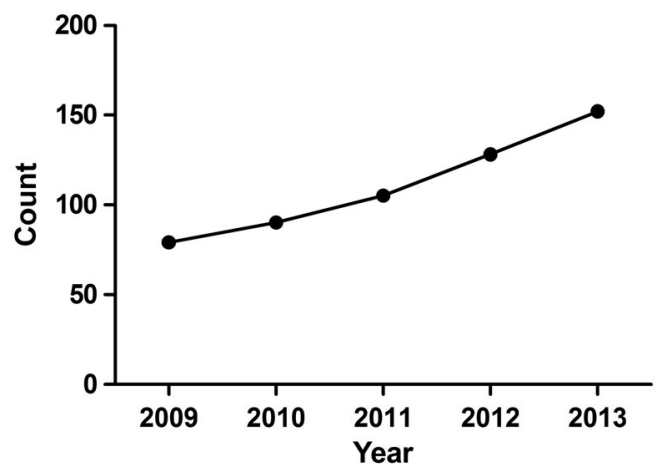

Figure 1 The annual count of TSCI patients during 2009-2013. cause $(13.2 \%)$. Furthermore, there were several unusual causes, such as injury in a collision with object(s) $(0.2 \%)$, machinery injuries $(0.9 \%)$, and hyper-traction, sports injuries, and other unusual injuries, accounting for $0.4 \%, 0.5 \%$ and $1.3 \%$, respectively.

\section{Level of injury}

The level of TSCI took on a bimodal distribution. The cervical region formed the first peak, accounting for more than $54 \%$ of total cases, especially C4-C6 $(36.8 \%)$, followed by a second peak for the thoracolumbar region, especially T11-L2 (30.3\%). Fig. 2 shows the distribution of the level of TSCI.

\section{ASIA grade and severity}

Percentages of ASIA A, B, C, and D injuries were 218 $(39.4 \%), 48(8.7 \%), 117(21.1 \%)$, and $171(30.8 \%)$ during this period (Fig. 3). The severity was classified as complete or incomplete injury according to the ASIA scale. Finally, the proportions of complete tetraplegia, incomplete tetraplegia, complete paraplegia 


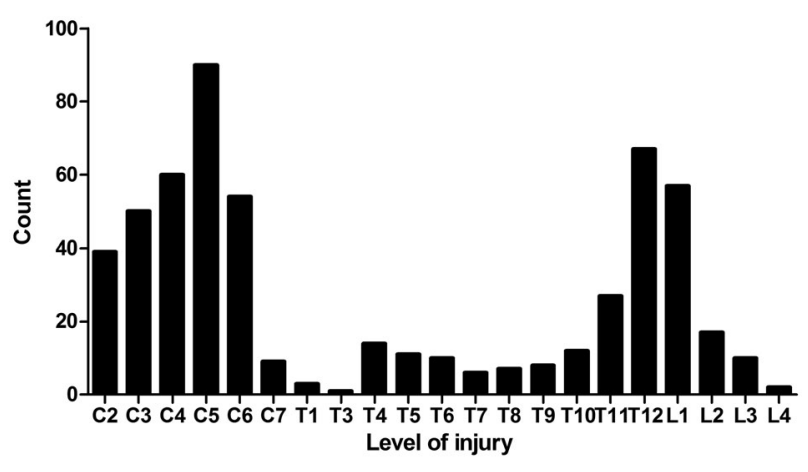

Figure 2 Distribution histogram of the injury level of the patients.

and incomplete paraplegia were $17.1 \%, 37.8 \%, 22.2 \%$, and $22.9 \%$, respectively.

\section{Death and the cause of death}

During hospitalization, eight patients died after being admitted; six were male and two were female. Half of these patients had been operated on. The oldest was only 64 years, followed by three patients (age $>50$ years) who had injuries from a high fall; the other four patients (age $<50$ years) were injured because of an object strike, an MVC, a low fall and a high fall. Six were injured in the cervical region, and two had an injury at the level of thoracolumbar region. Five died from multiple organ failure, two from pulmonary infections and one from respiratory failure.

\section{Concomitant injuries}

Among the 554 patients, the detailed review of medical records revealed that most patients with TSCI had common concomitant injuries, including fractures of the cervical, thoracic, or lumbar vertebrae, head injuries, limb or pelvic fractures, fractures of ribs, and

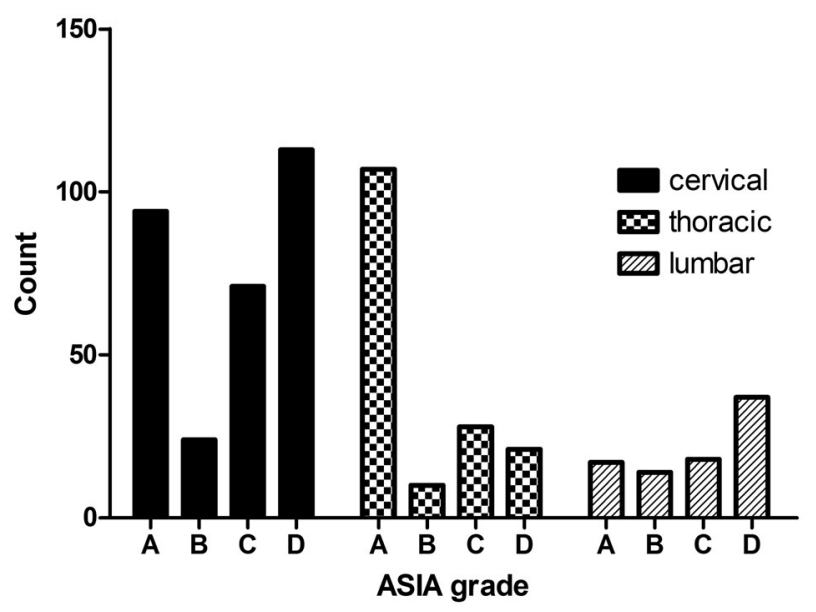

Figure 3 The count of patients in cervical, thoracic and lumbar segment according to the ASIA scale. other injuries, such as sternal fractures, hemopneumothorax, subarachnoid hemorrhage and lung contusion. In light of these results, we found that for patients with TSCI, falls, falling objects and MVCs were more likely to result in concomitant injuries compared with other causes. Because this research was about traumatic spinal cord injury, internal medicine diseases such as hypertension, diabetes, cardiac disease and anemia were not commonly reported during the period of study.

\section{Other results}

Within this period, the time from trauma to being admitted to the hospital ranged between 2 hours and 21 days. During this period, some patients might have received treatment in their local hospitals, and others might have been in an ambulance on the way to the hospital. Even worse, some patients might not have paid full attention to their trauma conditions and even stayed at home during this period. The length of hospital stay ranged between 1 and 219 days (mean: 28.3 days). And the hospital stay had a large range. As shown in the supplementary figure 1 , we calculated the numbers outliers, there were 39 numbers outliers (when the length of hospital stay were over 65 days). There were 465 patients $(83.9 \%)$ who received surgical treatment and 75 patients $(13.5 \%)$ who were conservatively treated, while 14 patients left the hospital against medical advice. In this study, $12.6 \%$ of the patients with TSCI experienced clinical complications. The four main complications were electrolyte disturbances $(1.9 \%)$ pulmonary infections $(7.0 \%)$, urinary tract infections $(1.3 \%)$, and bedsores $(1.9 \%)$. Some patients $(4.0 \%)$ experienced simultaneous multiple complications.

\section{Discussion}

To our knowledge, the current study is the first hospitalbased retrospective study on TSCI in Chongqing Province. Wang et al. performed a study describing the characteristics of traumatic cervical spinal fractures and risk factors for traumatic cervical SCI but did not include thoracic and lumbar SCI. ${ }^{13}$ Compared with other Chinese provinces, Chongqing has several unique characteristics. The short nickname for Chongqing is "mountain city," and it is one of the municipalities directly under the administration of the central government of the People's Republic of China, a wellknown economic and culture center in the upper Yangtze River region. There are many patients with TSCI in Chongqing; thus, understanding the epidemiological characteristics of TSCI in Chongqing is necessary for the optimal allocation of medical resources 
and to provide improved medical services to patients with TSCI.

In the present study, we described the epidemiological characteristics of TSCI over a 5-year period in Xinqiao Hospital, Chongqing, China. Most injuries occurred in the 31-45-year age-group, which is consistent with recent reports from research in Anhui ${ }^{10}$ and in Botswana $^{14}$ The reason why TSCI was likely to occur in the 31- to 45-year age group might be because Chongqing has many mountain roads; the rough terrain may result in more trips and falls, thereby increasing the risk of MVCs and low falls. Further, people (particularly in the 31- to 45-year age range) often perform income-related work outdoors to support their families. There is an additional heavy financial burden for laborers in the sandwich generation years, ${ }^{10}$ e.g. those with both aging parents and younger children.

In this investigation, the occupations of patients with TSCI included the unemployed $(24.2 \%)$, peasants $(31.9 \%)$, laborers $(23.6 \%)$, office-clerks $(3.6 \%)$, the retired $(2.0 \%)$, students $(1.8 \%)$, teachers $(0.4 \%)$, and others $(12.5 \%)$. Few studies have reported occupations of patients with TSCI. The results of occupational characteristics in the current study were the same as previously reported in Tianjin, ${ }^{11}$ Anhui, ${ }^{10}$ and India. ${ }^{15}$ In our results, the percentages of peasants and laborers were $31.9 \%$ (177) and $23.6 \%$ (131), respectively. This fact was mainly because the educational background of these patients was relatively low, thus limiting them to manual work and increased vulnerability to TSCI, which may explain the increased burden of TSCI in this occupational group.

The male/female ratio was 4.33:1 in the present research, which was similar to other studies, ${ }^{4,11,15-17}$ particularly similar to a systematic review. ${ }^{7}$ Similar to other regions, ${ }^{10,11}$ in Chongqing, the majority of women were also employed in occupations with low risk of TSCI, while high physical-risk behaviors are primarily performed by men.

The final data revealed that falls, including low fall and high fall, and MVCs were the two main causes of TSCI, which occurred at almost all ages. This finding is in accordance with a recent study in China. ${ }^{11}$ However, low fall was the main etiology of the age, ${ }^{17}$ and young people often suffered from TSCI because of high fall and MVCs, ${ }^{18}$ depending on their different activities and work environment. Another reason why MVCs were the second cause of TSCI was that more people may have cars and be more likely to use cars as a main mode of transportation. Those individuals traveling by car may be more susceptible to TSCI, should an accident occur.
The most common levels of injury observed in the present study were at C4-C6 and T11-L2, which took on a bimodal distribution. This finding demonstrated that the probability of cervical and thoracolumbar spinal cord injuries was very high, which was similar to other reports. ${ }^{10,19}$ The structures of the spinal column and mechanisms of fractures determined the injury severity and level. The cervical spine proved to be more vulnerable than other vertebrae. ${ }^{20}$

During this period, percentages of ASIA A, B, C, and $\mathrm{D}$ injuries were $39.4 \%, 8.7 \%, 21.1 \%$, and $30.8 \%$, respectively, and ASIA A and D injuries comprised the majority of TSCI cases. Increased attention should be paid to ASIA A patients when they encounter TSCI, as they are more prone to suffer from depressive disorders $^{21}$ and suicide. ${ }^{22}$

Several limitations need to be considered in the current study: (1) there was a lack of information on the training and qualifications of the individuals identifying patients with TSCI in Chongqing, even in China. Thus, sufficient available TSCI epidemiological research was not found in China. It is of great importance to implement a systematic TSCI registry center in China in future. (2) This study was a hospital-based descriptive study about TSCI, which identified only a small part of all patients with TSCI in Chongqing; thus, the overall incidence rate could not be calculated. (3) The identification of patients with TSCI was based on a review of hospital medical records by two reviewers. The reviewers used mostly consistent but not completely identical identification criteria. (4) Surgeons or registrars may not have recorded the information accurately at the time, and because hospital medical records were registered by different doctors, there may be some potential errors. (5) We could not obtain detailed occupational information on those who were unemployed, perhaps because the registrars neglected to perform such inquiries when the patients registered at the hospital. (6) Patients with only minor motor and sensory dysfunctions were not always diagnosed properly. (7) Patients died prior to getting to the hospital; thus, diagnosis was uncertain and complete medical records were not included, which could lead to an underestimation of the incidence rate.

\section{Conclusion}

Generally, although there were several limitations, our study, a retrospective study of the characteristics of patients with TSCI admitted to Xinqiao Hospital from 2009 to 2013, was similar to other related research about the epidemiological characteristics of TSCI. The results revealed that the proportion of males was 
higher, falls and MVCs were the two main causes, and the mean age of patients with TSCI was older than in other countries. The most frequent high-risk occupations reported in patients with TSCI were peasants and laborers. All of these results prompted the conclusion that preventive approaches should be based on the characteristics of the different types of patients with TSCI and focused on high-risk groups, such as laborers, peasants and the aged.

\section{References}

1 French DD, Campbell RR, Sabharwal S, Nelson AL, Palacios PA, Gavin-Dreschnack D. Health care costs for patients with chronic spinal cord injury in the Veterans Health Administration. J Spinal Cord Med 2007;30(5):477-81.

2 Cripps RA, Lee BB, Wing P, Weerts E, Mackay J, Brown D. A global map for traumatic spinal cord injury epidemiology: towards a living data repository for injury prevention. Spinal Cord 2011;49(4):493-501.

3 Wyndaele M, Wyndaele JJ. Incidence, prevalence and epidemiology of spinal cord injury: what learns a worldwide literature survey? Spinal Cord 2006;44 (9):523-9.

4 Bjornshave Noe B, Mikkelsen EM, Hansen RM, Thygesen M, Hagen EM. Incidence of traumatic spinal cord injury in Denmark, 1990-2012: a hospital-based study. Spinal Cord 2015; $53(6): 436-40$

5 Koskinen EA, Alen M, Vaarala EM, Rellman J, Kallinen M, Vainionpaa A. Centralized spinal cord injury care in Finland: unveiling the hidden incidence of traumatic injuries. Spinal Cord 2014;52(10):779-84.

6 Robert AA, Zamzami MM. Traumatic spinal cord injury in Saudi Arabia: a review of the literature. Pan Afr Med J 2013;16:104.

7 Rahimi-Movaghar V, Sayyah MK, Akbari H, Khorramirouz R, Rasouli MR, Moradi-Lakeh M, et al. Epidemiology of traumatic spinal cord injury in developing countries: a systematic review. Neuroepidemiology 2013;41(2):65-85.

8 Ning GZ, Yu TQ, Feng SQ, Zhou XH, Ban DX, Liu Y et al, Epidemiology of traumatic spinal cord injury in Tianjin, China. Spinal Cord 2011;49(3):386-90.

9 Li J, Liu G, Zheng Y, Hao C, Zhang Y, Wei B et al, The epidemiological survey of acute traumatic spinal cord injury (ATSCI) of 2002 in Beijing municipality. Spinal Cord 2011;49(7):777-82.
10 Wang HF, Yin ZS, Chen Y, Duan ZH, Hou S, He J. Epidemiological features of traumatic spinal cord injury in Anhui Province, China. Spinal Cord 2013;51(1):20-2.

11 Feng HY, Ning GZ, Feng SQ, Yu TQ, Zhou HX. Epidemiological profile of 239 traumatic spinal cord injury cases over a period of 12 years in Tianjin, China. J Spinal Cord Med 2011;34(4): 388-94.

12 Knutsdottir S, Thorisdottir H, Sigvaldason K, Jonsson H Jr, Bjornsson A, Ingvarsson P. Epidemiology of traumatic spinal cord injuries in Iceland from 1975 to 2009. Spinal Cord 2012; 50(2):123-6.

13 Wang H, Xiang Q, Li C, Zhou Y. Epidemiology of traumatic cervical spinal fractures and risk factors for traumatic cervical spinal cord injury in China. J Spinal Disord Tech 2013;26(8): E306-13.

14 Lofvenmark I, Norrbrink C, Nilsson-Wikmar L, Hultling C, Chakandinakira S, Hasselberg M. Traumatic spinal cord injury in Botswana: characteristics, aetiology and mortality. Spinal Cord 2015;53(2):150-4.

15 Mathur N, Jain S, Kumar N, Srivastava A, Purohit N, Patni A. Spinal cord injury: scenario in an Indian state. Spinal Cord 2015; 53(5):349-52.

16 Equebal A, Anwer S, Kumar R. The prevalence and impact of age and gender on rehabilitation outcomes in spinal cord injury in India: a retrospective pilot study. Spinal Cord 2013;51(5): 409-12.

17 Thompson C, Mutch J, Parent S, Mac-Thiong JM. The changing demographics of traumatic spinal cord injury: an 11-year study of 831 patients. J Spinal Cord Med 2015;38(2):214-23.

18 New PW, Baxter D, Farry A, Noonan VK. Estimating the incidence and prevalence of traumatic spinal cord injury in australia. Arch Phys Med Rehabil 2015;96(1):76-83.

19 Fredo HL, Rizvi SA, Lied B, Ronning P, Helseth E. The epidemiology of traumatic cervical spine fractures: a prospective population study from Norway. Scand J Trauma Resusc Emerg Med. 2012;20:85.

20 Lin CY, Wright J, Bushnik T, Shem K. Traumatic spinal cord injuries in horseback riding: a 35-year review. Am J Sports Med 2011; 39(11):2441-6.

21 Williams RT, Wilson CS, Heinemann AW, Lazowski LE, Fann JR, Bombardier CH. Identifying depression severity risk factors in persons with traumatic spinal cord injury. Rehabil Psychol 2014; 59(1):50-6.

22 Thietje R, Pouw MH, Schulz AP, Kienast B, Hirschfeld S. Mortality in patients with traumatic spinal cord injury: descriptive analysis of 62 deceased subjects. J Spinal Cord Med 2011;34(5): $482-7$. 